the atmosphere over the northern hemisphere takes the quantity of natural particles as a steady 690 million tons (110 million tons from oceans and 580 million tons from land surfaces); added to this is a man-made contribution, put at 480 million tons today, compared with 120 million tons back in 1880 and a predicted 760 million tons by the year 2000. Amidst the justifiable concern about possible implications of this trend, there has been much jumping to conclusions and a general tendency to overlook, or ignore, the incidence of natural fluctuations and trends in climate.

Excessively confident voices among scientists in the United States and Russia, and some leaders of opinion elsewhere, have proclaimed in recent years that it is the increasing incidence of man-made dust in the atmosphere that accounts for the change in climatic trends since 1950. Before about that time a global warming was in progress which could be traced back as early as the beginnings of the industrial revolution and which had, in the same circles, been perhaps too hastily equated with the effect of increasing carbon dioxide as a trap for the outgoing terrestrial radiation. The argument that man-made dust is now more effective than the (still increasing) quantity of carbon dioxide has been drawn from the demonstrated effect of volcanic dust veils in the stratosphere in lowering the overall average temperature near the surface of the Earth by up to $1^{\circ} \mathrm{C}$ for a year or two after the great eruptions for which adequate observations exist.

But the analogy is largely false and, at best, of very limited value. Although even the greatest volcanic eruptions are estimated to put only from 1 million to 100 million tons of finely divided particles into the atmosphere, typical residence times in the stratosphere are several years. The mean residence time for particulate matter in the lower atmosphere is about 10 days, and it is then washed back to the Earth with the rain and snow. Moreover, solar radiation absorbed by matter suspended in the commonly soiled atmosphere in the lowest 1 to $3 \mathrm{~km}$ must be a more effective contribution to the energy budget at the Earth's surface than that which is intercepted by volcanic dust in the stratosphere. It does not take much thought to add to this list of $a$ priori differences.

There is a great need for concerted efforts to establish the nature and magnitude of the meteorological and climatic effects of dust and other types of pollution in the lower atmosphere. Much survey work will be needed and many types of new observational techniques may be useful in supplying quantitative evidence of the facts.-

H. H. L.

\section{Consequences and Truth}

THE scientist feels that there is order in nature, if only it can be found. Indeed, such feelings have been the basis for most of scientific research, and results from all disciplines support this view. Thus the scientist is always looking for correlations.

Earth scientists are no exception, but they work in a field where proof, in the mathematician's sense of the word, is hard to find, or may be impossible to find because of the problems of observing geological processes at work. Earth scientists are normally presented with the results of a process that may have lasted tens or hundreds of millions of years. Their job is to try to untangle the usually complex nature of the process from incomplete and often apparently conflicting evidence. This may perhaps be looked on as the reverse of a chemist's function, which is to control carefully the course of an experiment to produce an unknown or at best merely predicted result.

The concept of plate tectonics is a fine example of a unifying theory on a global scale which attempts to account for many different features on and in the Earth. Another such hypothesis was the Flood. It is perhaps unfair to compare the two, but many eminent scientists have spent much time looking for evidence in support of both of them. Both, in their time, seem to satisfy the scientist's craving for order in nature. It should perhaps be made clear that there are now some rather sinuous drawbacks to the Flood theory; it is amazing how one unifying theory after another has gone the way of the world, but this should come as no surprise and should be ascribed to progress.

According to plate tectonics the lithosphere (the outer $200 \mathrm{~km}$ or so of the Earth) moves about as a number of fairly rigid plates. In some places, for example along the line of the Mid-Atlantic Ridge, the plates are separating, because of the addition of material from below into the crack. In other places lithospheric material is being forced under the adjacent plate; the Earth is probably not expanding. If this concept of moving plates is accepted then many consequences follow, and it is these that many Earth scientists are engaged in examining at the moment, although, of course, the consequences do not necessarily mean that the hypothesis is the truth (see Cobbing and Pitcher, Nature Physical Science, 240, 51 ; 1972).

This is the bandwagon, a very good one in my opinion, which is on the move in geology, providing the most intense stimulus to new ideas that the subject has ever had. It is thus very fortunate that the new exhibition of basic geological processes at the Geological Museum in South Kensington has appeared now, and not ten years ago.

On page 338 of this issue of Nature, P. R. Vogt of the US Naval Oceanographic Office examines one of the facets of the plate tectonic hypothesis, that of the possible effects of synchroneity in mantle plumes. Mantle plumes are more or less linear currents of material brought up from below the lithosphere, and it is believed that they may be one of the driving forces in plate movement. Vogt has examined the products (lavas) of two such plumes, in the Hawaiian chain and in Iceland, in detail and suggests that there is good evidence for synchronous events not only in these two plumes but also in a dozen more. $\mathrm{He}$ then attempts to relate this to other phenomena on a global scale (sedimentation rates in the oceans, magnetic reversals and biological extinctions). The relation between mantle plume activity and the "elimination of numerous genera" is particularly interesting. Vogt rehabilitates Professor Preston Cloud's argument that because marine or brackish water organisms are sensitive to the chemistry of their environment, perhaps the demise on a world-wide scale of certain genera can be attributed to changes in the concentration of trace metals in sea water.

Earth scientists are thus in their usual geological dilemma. They can observe the consequences, but what is the truth?-A. C. D. 